\title{
Computation of continuous and piecewise affine Lyapunov functions by numerical approximations of the Massera construction
}

\author{
Jóhann Björnsson ${ }^{1}$, Peter Giesl ${ }^{2}$, Sigurdur Hafstein ${ }^{1}$, Christopher M. Kellett ${ }^{3}$, and Huijuan $\mathrm{Li}^{4}$
}

\begin{abstract}
The numerical construction of Lyapunov functions provides useful information on system behavior. In the Continuous and Piecewise Affine (CPA) method, linear programming is used to parameterize a CPA Lyapunov function for continuous nonlinear systems. This method is relatively slow due to the linear program that has to be solved. A recent proposal was to parameterize the CPA Lyapunov function based on a Lyapunov function in a converse Lyapunov theorem by Yoshizawa. In this paper we propose parameterizing CPA Lyapunov functions using a Lyapunov function construction in a classic converse Lyapunov theorem by Massera. We provide the theory for such a parameterization and present several examples to illustrate the utility of this approach.
\end{abstract}

\section{INTRODUCTION}

Let $K \subset \mathbb{R}^{n}$ be a compact neighborhood of the origin and consider a $C^{2}$ vector field $f: K \rightarrow \mathbb{R}^{n}$. We consider a dynamical system

$$
\dot{\mathbf{x}}=f(\mathbf{x})
$$

where the origin is an asymptotically stable equilibrium point. As the general reader is well aware of, it can be difficult to find a particular solution to such a system, that is for a given $\mathbf{x} \in K$ and an interval $I \subset \mathbb{R}_{>0}$ find a function $\phi: I \times \mathbb{R}^{n} \rightarrow \mathbb{R}^{n}$ such that $\frac{d}{d t} \phi(t, \mathbf{x})=$ $f(\phi(t, \mathbf{x})), \phi(0, \mathbf{x})=\mathbf{x}$. Our goal then instead is to construct a continuous Lyapunov function for the system, that is a positive definite continuous function $V: K \rightarrow \mathbb{R}$ such that for every solution $\phi$ of $\frac{d}{d t} \phi(t, \mathbf{x})=f(\phi(t, \mathbf{x}))$ on $K \backslash\{\mathbf{0}\}$ we have for every $t_{0}, t_{1} \in I$ that $t_{0}<t_{1}$ implies $V\left(\phi\left(t_{0}\right)\right)<V\left(\phi\left(t_{1}\right)\right)$. It is well known that this condition follows from a Lipschitz $V$ such that

$$
V^{+}(\mathbf{x}):=\limsup _{h \rightarrow 0+} \frac{V(\mathbf{x}+h f(\mathbf{x}))-V(\mathbf{x})}{h}
$$

is negative definite on $K^{\circ}$. Note that $V^{+}(\mathbf{x})$ is sometimes referred to as the orbital derivative of $V$ at $\mathbf{x}$ with respect to $f$; or simply the orbital derivative of $V$.

In general, constructing a Lyapunov function for (I.1) is as difficult as finding solutions to system (I.1). However, once

\footnotetext{
${ }^{1}$ J. Björnsson (johannsb13@ru.is) and S. Hafstein (sigurdurh@ru.is) are with the School of Science and Engineering, Reykjavik University, Iceland. Björnsson is supported by The Icelandic Research Fund, grant nr. 130677052. ${ }^{2} \mathrm{P}$. Giesl is with the Department of Mathematics, University of Sussex, UK. ${ }^{3}$ C.M. Kellett (Chris.Kellett@ newcastle.edu.au) is with the School of Electrical Engineering and Computer Science, University of Newcastle, Callaghan, New South Wales, Australia. Kellett is supported by ARC Future Fellowship FT1101000746 and by the Alexander von Humboldt Foundation. ${ }^{4} \mathrm{H}$. Li (Huijuan.Li@uni-bayreuth.de) is with the Department of Mathematics, University of Bayreuth, Germany. $\mathrm{Li}$ is supported by the EU Initial Training Network "Sensitivity Analysis for Deterministic Controller Design-SADCO".
}

constructed on a specific domain, a Lyapunov function gives a good idea of the behavior of all solutions in that domain.

There have been numerous proposals of how to compute Lyapunov functions numerically for nonlinear systems. For example, collocation methods were proposed in [11], [3], [21], graph theoretic methods in [2], [12], semidefinite optimization for sum-of-squares polynomials (SOS method) [17], [18], [19], as well as automated algebraic methods [20], [22].

In [15] a method was proposed for constructing a Lyapunov function by solving linear inequalities on a finite set of points in $K$, called a vertex set for $K$, which in turn determine a unique continuous piecewise affine (CPA) Lyapunov function on the whole of $K$. This method is referred to as the CPA method. The CPA method has been improved [7] and extended to different kinds of systems [9], [1], [5], [6].

The method presented here is a simple modification of the CPA method where, instead of using linear programming to determine the values at the points of the vertex set, we use a function construction from a classical converse Lyapunov theorem developed by Massera [16] to determine the values. This is similar to the procedure in [10] where a construction due to Yoshizawa [24] was used.

This paper is organized as follows. In Section II we give a brief summary of the CPA method and describe the converse Lyapunov theorem that provides the function we use to define a CPA function. In Section III we present four numerical examples illustrating the utility of our proposed construction.

We denote the positive integers by $\mathbb{N}$ and the strictly positive real numbers by $\mathbb{R}_{>0}$. For a vector $\mathbf{x}=\left[x_{1}, \ldots, x_{n}\right]^{T} \in$ $\mathbb{R}^{n}$ we define the norms $\|\mathbf{x}\|_{p}=\left(\sum_{i=1}^{n}\left|x_{i}\right|^{p}\right)^{\frac{1}{p}}$ for $p \geq 1$ as is usual, and denote for a set $A \subseteq \mathbb{R}^{n}$ its diameter by $\operatorname{diam}_{2}(A):=\sup _{\mathbf{x}, \mathbf{y} \in A}\|\mathbf{x}-\mathbf{y}\|_{2}$. We denote the open ball in $\mathbb{R}^{n}$ of radius $r>0$ centered at the origin by

$$
B_{r}:=\left\{\mathbf{x} \in \mathbb{R}^{n}:\|\mathbf{x}\|_{2}<r\right\} .
$$

\section{THEORY}

We start by giving a summary of the CPA method as presented in [9]. We define a grid on $K \subset \mathbb{R}^{n}$ and calculate for each point $\mathrm{x}$ in the grid the value that $V$ is to take at $\mathrm{x}$. The values for $V$ on the grid can then be used to extend $V$ to all of $K$ such that $V$ is a continuous piecewise affine Lyapunov function on $K$. In this section we shall provide the details of this construction.

Let $\mathbf{x}_{0}, \ldots, \mathbf{x}_{k}$ be a collection of linearly affine independent points in $\mathbb{R}^{n}$, that is $\sum_{i=0}^{k} c_{i}\left(\mathbf{x}_{i}-\mathbf{x}_{0}\right)=\mathbf{0}$ 
implies that $c_{i}=0$ for $i=1, \ldots, k$. The convex combination of $\mathbf{x}_{0}, \ldots, \mathbf{x}_{k}$, that is the set $\operatorname{co}\left\{\mathbf{x}_{0}, \ldots, \mathbf{x}_{k}\right\}:=$ $\left\{\sum_{i=0}^{k} c_{i} \mathbf{x}_{i} \mid\right.$ all $c_{i} \geq 0$ and $\left.\sum_{i=0}^{k} c_{i}=1\right\}$, is a $k$-simplex in $\mathbb{R}^{n}$.

Let $\mathcal{T}=\left\{T_{1}, \ldots, T_{m}\right\}$ be a set of k-simplices such that $K=\cup_{i=1}^{m} T_{i}$. Suppose further that for every $i, j$ such that $i \neq$ $j$ we have that $T_{i} \cap T_{j}=\varnothing$ or that $T_{i} \cap T_{j}$ is a h-simplex with $h<k$. We then say that $\mathcal{T}$ is a triangulation of $K$. We further define $\mathcal{V}_{\mathcal{T}}:=\left\{\mathbf{x} \in \mathbb{R}^{n} \mid \mathbf{x}\right.$ is a vertex of a simplex in $\left.\mathcal{T}\right\}$ and call $\mathcal{V}_{\mathcal{T}}$ the vertex set for the triangulation.

For a given triangulation $\mathcal{T}=\left\{T_{1}, \ldots, T_{m}\right\}$ and a function $V_{0}: \mathcal{V}_{\mathcal{T}} \rightarrow \mathbb{R}$ we can now uniquely extend $V_{0}$ to a continuous piecewise affine function $V: K \rightarrow \mathbb{R}$ that is $C^{\infty}$ in the interior of each simplex $T_{i}$. More specifically:

i) If $\mathbf{x} \in \mathcal{V}_{\mathcal{T}}$ then $V(\mathbf{x})=V_{0}(\mathbf{x})$.

ii) For every $T_{i} \in \mathcal{T}$ there exists a linear mapping $A_{i}$ : $\mathbb{R}^{n} \rightarrow \mathbb{R}$ and $b_{i} \in \mathbb{R}$ such that $V(\mathbf{x})=A_{i}(\mathbf{x})+b_{i}$ for all $\mathbf{x} \in T_{i}$ and such that $V$ is continuous on $K$.

We are interested in systems with an asymptotically stable equilibrium at the origin and consequently in Lyapunov functions with a minimum at the origin. Since a local minimum for a CPA function can only be attained at a vertex, we insist that the origin be a vertex of our triangulation. For a given triangulation $\mathcal{T}$ of $K$ we denote the collection of all continuous piecewise affine functions as described above by $\mathrm{CPA}[\mathcal{T}]$ and identify each such function with the corresponding function $V_{0}: \mathcal{V}_{\mathcal{T}} \rightarrow \mathbb{R}$ by $V \sim\left(V_{0}(\mathbf{x})\right)_{\mathbf{x} \in \mathcal{V}_{\mathcal{T}}}$.

The following theorem from [9] states that in order to construct a Lyapunov function for (I.1) it is sufficient to determine a function $V_{0}: \mathcal{V}_{\mathcal{T}} \rightarrow \mathbb{R}$ which satisfies certain inequalities at each vertex $\mathrm{x} \in \mathcal{V}_{\mathcal{T}}$, for then the CPA interpolation of $V_{0}$ on $K$ is a CPA Lyapunov function for (I.1) on the whole of $K$.

Theorem 2.1: Consider the system (I.1) with triangulation $\mathcal{T}=\left\{T_{1}, \ldots, T_{m}\right\}$. Let $V \sim\left(V_{0}(\mathbf{x})\right)_{\mathbf{x} \in \mathcal{V}_{\mathcal{T}}} \in \mathrm{CPA}[\mathcal{T}]$. For each $T_{i} \in \mathcal{T}$ define the constants $h_{i}:=\operatorname{diam}_{2}\left(T_{i}\right)$ and

$$
\begin{aligned}
E_{i} & :=\frac{n M_{i}}{2} h_{i}^{2}, \text { where } \\
M_{i} & \geq \max _{m, r, s=1,2, \ldots, n} \max _{\mathbf{z} \in T_{i}}\left|\frac{\partial^{2} f_{m}}{\partial x_{r} \partial x_{s}}(\mathbf{z})\right| .
\end{aligned}
$$

Assume that for a simplex $T_{i}=\operatorname{co}\left\{\mathbf{x}_{0}, \mathbf{x}_{1}, \ldots, \mathbf{x}_{k}\right\} \in \mathcal{T}$ the inequality

$$
\nabla V_{i} \cdot f\left(\mathbf{x}_{j}\right)+E_{i}\left\|\nabla V_{i}\right\|_{1}<0
$$

holds true for every vertex $\mathbf{x}_{j} \in T_{i}$. Then

$$
\nabla V_{i} \cdot f(\mathbf{x})<0 \text { for all } \mathbf{x} \in T_{i}
$$

If the inequality (II.2) holds true for $T_{i_{1}}, T_{i_{2}}, \ldots, T_{i_{m}}$, we have $V^{+}(\mathbf{x})<0$ for all $\mathbf{x} \in\left(\cup_{j=1}^{m} T_{i_{j}}\right)^{\circ}$.

In [9] the values of $V_{0}$ at the vertex points are determined by linear programming. Here we will propose a different and a much faster method to calculate the vertex values similar to the one in [10]. In [16] Massera proved a converse Lyapunov theorem for systems with an asymptotically stable equilibrium at the origin by constructing a Lyapunov function based on the integral of a nonlinear scaling of the norm of the state trajectory. While Massera's construction is applicable to asymptotic stability, for simplicity we here assume exponential stability. The following formulation is a slightly simplified version of the one contained in [14].

Theorem 2.2: Consider a dynamical system $\dot{\mathbf{x}}=f(\mathbf{x})$ where $f$ is $C^{2}$ on $B_{r} \subset \mathbb{R}^{n}$ for some $r \in \mathbb{R}_{>0}$ and suppose that $\mathbf{0}$ is an exponentially stable equilibrium point for the system, that is there exist constants $c, \lambda \in \mathbb{R}_{>0}$ such that $\|\phi(t, \mathbf{x})\| \leq c\|\mathbf{x}\| e^{-\lambda t}$ for all $t \geq 0$ such that $\mathbf{x} \in B_{r}$. Then there exists a constant $N$ such that

$$
V(\mathbf{x}):=\int_{0}^{N}\|\phi(\tau, \mathbf{x})\|_{2} d \tau .
$$

is a Lyapunov function for the system on $B_{r}$.

As indicated in [14] the constant $N$ can be taken to be $N=\frac{\ln \left(2 k^{2}\right)}{2 \lambda}$. However, since it can be difficult to determine $\lambda$ and $k$ for specific systems this equation is of limited practical value. It is also worth noting that the function constructed in Theorem 2.2 inherits the smoothness attributes of $f$, essentially because $\phi$ does, so we have $V \in C^{2}\left(B_{r} \backslash\{\mathbf{0}\}\right)$.

To generalize the above to asymptotically stable systems we can take a nonlinear scaling of the norm of the state trajectory obtained from a class- $\mathcal{K} \mathcal{L}$ asymptotic stability estimate and Sontag's Lemma on $\mathcal{K} \mathcal{L}$-estimates [23, Proposition 7] which provides an exponentially decreasing in time upper bound for the asymptotic stability estimate (see [13] for the definition of class- $\mathcal{K} \mathcal{L})$.

Our modification of the CPA method is now the following:

1) For a given compact neighborhood $K \subset \mathbb{R}^{n}$ of the origin, define a sequence $\left(\mathcal{T}_{j}\right)_{j \in \mathbb{N}}$ of triangulations of $K$ that have uniformly bounded degeneracy (for details, cf. [1], [10]) and such that

$$
\lim _{j \rightarrow \infty}\left(\max _{T \in \mathcal{T}_{j}} \operatorname{diam}_{2}(T)\right)=0 .
$$

2) Fix an increasing function $g: \mathbb{N} \rightarrow \mathbb{R}_{>0}$ with $\lim _{j \rightarrow \infty} g(j)=\infty$.

3) Set $j=1$.

4) Compute

$$
V_{0}^{j}(\mathbf{x})=\int_{0}^{g(j)}\|\phi(\tau, \mathbf{x})\|_{2} d \tau
$$

at the vertices $\mathcal{V}_{\mathcal{T}_{j}}$ and by a convex interpolation construct a CPA function $V^{j}(\mathbf{x})$ on $K$.

5) Check inequality (II.2) at each vertex to determine where the CPA function $V^{j}(\mathbf{x})$ is a true Lyapunov function for (I.1). If a finer result is required, increment $j$ and return to Step 4. Otherwise stop.

By our choice of $g$ it is obvious that $g(j)>\frac{\ln \left(2 k^{2}\right)}{2 \lambda}$ in the long run, and in [8] and [10] it is demonstrated that there exists a value $\delta>0$ such that if $\max _{T \in \mathcal{T}} \operatorname{diam}_{2}(T)<\delta$ then the Lyapunov function constructed by the CPA method in fact satisfies inequality (II.2) at every vertex point. Therefore, if $K$ is in the basin of attraction of the equilibrium and given a priori an arbitrary small $\varepsilon$-ball $B_{\varepsilon}$ centered at the 
origin, the functions $V^{j}$ will be true Lyapunov functions for the system on $K \backslash B_{\varepsilon}$ for all large enough $j$. For all but the most simple systems one has to approximate the values of $V_{0}$ numerically. It is a matter of ongoing research how this affects the inequalities at the vertices, but our numerical examples are promising.

\section{EXAMPLES}

In this section we shall present numerical examples that demonstrate the effectiveness of our modified CPA method. In what follows we use the classical Runge-Kutta method RK4 to estimate $\phi(t, \mathbf{x})$. Across the examples, various stepsizes $\Delta t$ for RK4 were used and for Examples 1-3 $\Delta t=0.01$ was found to be sufficiently small. For Example 4 we used a smaller step-size of $\Delta t=0.001$ for better results.

In the following examples we set $g(1)=100 \cdot \Delta t, S_{1}=$ 800 and define recursively $g(j+1)=2 \cdot g(j), S_{j+1}=$ $4 \cdot S_{j}$, where $S_{j}$ denotes the number of simplices in our triangulation in the $j$-th step of the algorithm. In other words, at each iteration of the algorithm we double the upper limit of integration in (II.4) and have a fourfold increase in the number of simplices.

In the following figures we plot the calculated CPA function at a specific iteration of the algorithm and mark with * the simplices in which the computed CPA function fails to have a negative orbital derivative. It is also worth noting that all the functions we have calculated have been normalized such that the maximum value of the CPA Lyapunov function on its domain of definition is 1 .

\section{Example 1}

Our first example is the linear system

$$
\dot{\mathbf{x}}=A \mathbf{x}=\left[\begin{array}{cc}
1 & 1 \\
-5 & -3
\end{array}\right] \mathbf{x} .
$$

Since the eigenvalues of $A$ are $-1+i$ and $-1-i$ this system has an exponentially stable equilibrium at the origin. We now utilize the proposed modified CPA method above to calculate a Lyapunov function for the system on the unit disc, that is on $B_{1}$. We plot the function given by the first two iterations of the algorithm in Figures 1 and 2. Note the rapid convergence towards a true Lyapunov function in the whole domain for the system. In Figure 3 we plot some level curves for the CPA Lyapunov function obtained on the third iteration. In Table I we give two measures for the quality of the CPA Lyapunov functions computed in the different iterations.

\section{Example 2}

Our next example is the non-linear system

$$
\begin{aligned}
& \dot{x}_{1}=-x_{2}-\left(1-x_{1}^{2}-x_{2}^{2}\right) x_{1} \\
& \dot{x}_{2}=x_{1}-\left(1-x_{1}^{2}-x_{2}^{2}\right) x_{2} .
\end{aligned}
$$

By using polar coordinates or by linearizing one can demonstrate that the origin is exponentially stable. It is also simple to show that the unit circle is a recurrent set in this example, therefore it is wise to utilize our method on

\begin{tabular}{|c|c|c|}
\hline Iteration & $\begin{array}{c}\text { Percentage of Simplices } \\
\text { with } D^{+} V(\mathbf{x})<0\end{array}$ & $\begin{array}{c}\text { Radius }(R) \text { Outside } \\
\text { Which } D^{+} V<0\end{array}$ \\
\hline 1 & $91.750 \%$ & 1 \\
2 & $98.813 \%$ & $6.25 \cdot 10^{-2}$ \\
3 & $99.719 \%$ & $1.563 \cdot 10^{-2}$ \\
4 & $99.930 \%$ & $3.91 \cdot 10^{-3}$ \\
5 & $99.982 \%$ & $9.8 \cdot 10^{-4}$ \\
6 & $99.996 \%$ & $2.4 \cdot 10^{-4}$ \\
\hline
\end{tabular}

TABLE I

TWO DIFFERENT MEASURES FOR THE QUALITY OF THE COMPUTED CPA LYAPUNOV FUNCTIONS FOR THE DIFFERENT ITERATIONS. IN COLUMN 2 WE GIVE THE PERCENT OF THE NUMBER OF TRIANGLES $T_{i} \in \mathcal{T}$, WHERE THE ORBITAL DERIVATIVE $D^{+} V$ IS NEGATIVE AND IN COLUMN 3 WE GIVE THE RADIUS $R$ OF A BALL $B_{R}$, OUTSIDE OF WHICH THE ORBITAL DERIVATIVE IS NEGATIVE.

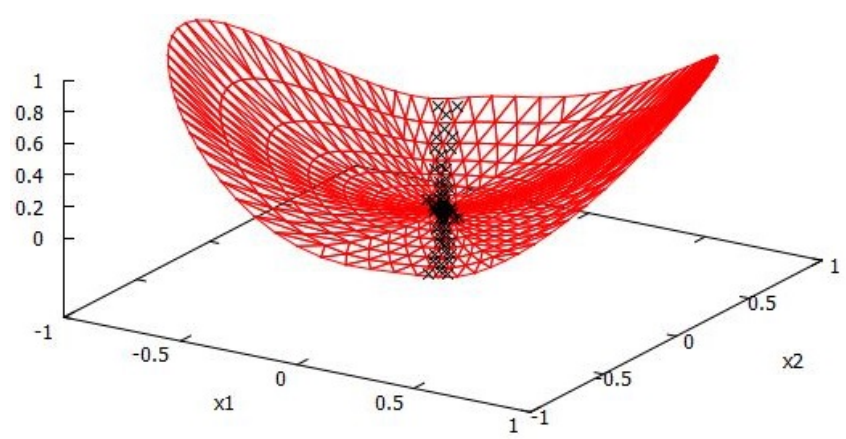

Fig. 1.

First iteration of the algorithm for the system in Example 1. We obtain a CPA Lyapunov function for the system with negative orbital derivative in 734 of the 800 simplices in the triangulation.

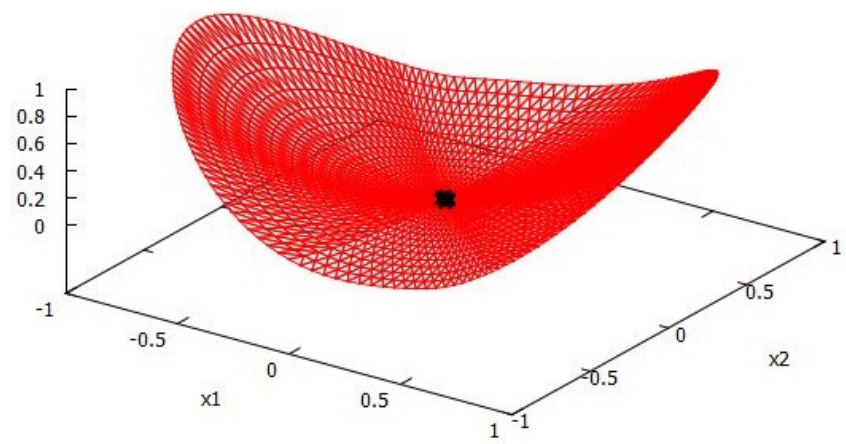

Fig. 2.

Second iteration of the algorithm for the system in Example 1. We obtain a CPA Lyapunov function for the system with negative orbital derivative in 3162 of the 3200 simplices in the triangulation. 


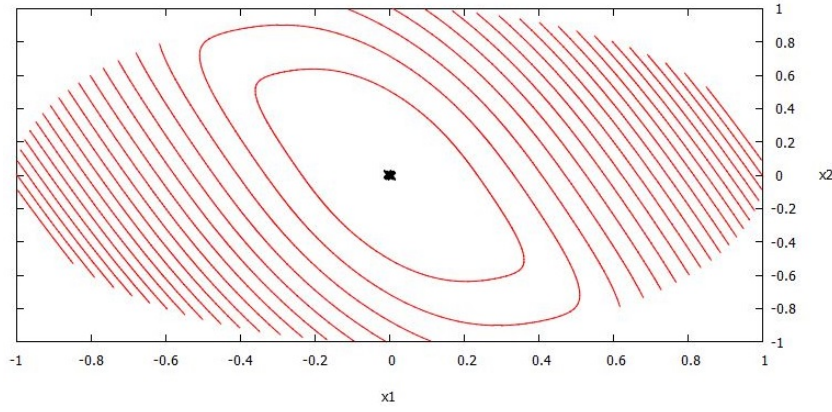

Fig. 3.

Some level curves of the CPA Lyapunov function from the third iteration for Example 1.

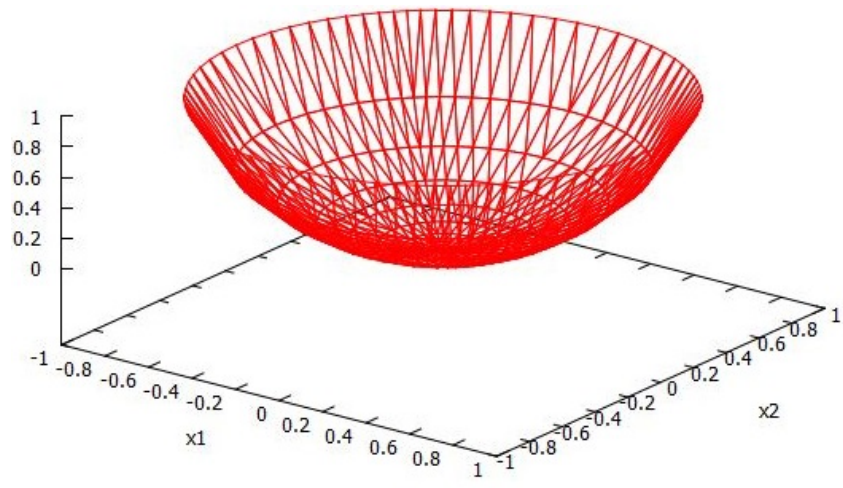

Fig. 4.

First iteration of the algorithm for the system in Example

2. We obtain a CPA Lyapunov function for the system with negative orbital derivative in all of the simplices in the triangulation. Therefore no further iterations are required.

a set slightly smaller than $B_{1}$. We choose the set $B_{0.95}$. In this case we obtain a Lyapunov function on the first iteration, so further iterations are unnecessary. The resulting CPA Lyapunov function is displayed in Figure 4. By using a slightly stricter version of Theorem 2.1 (cf. [4, Theorem 2.6]), we can even assure that the CPA Lyapunov function has a negative orbital derivative on its entire domain, even in a neighborhood of the origin.

In Figure 5 we plot some of its level curves.

\section{Example 3}

Next up is a torture test for the method. We consider the following non-linear system which we examine on the unit disc.

$$
\begin{aligned}
& \dot{x}_{1}=-x_{2}-2 x_{1}\left(x_{1}^{2}+x_{2}^{2}\right) \\
& \dot{x}_{2}=x_{1}-2 x_{2}\left(x_{1}^{2}+x_{2}^{2}\right) .
\end{aligned}
$$

Note the equilibrium at the origin is asymptotically stable but not exponentially stable. Further, solutions in its domain of attraction spiral around the equilibrium and converge very slowly to the equilibrium. As expected, the convergence of

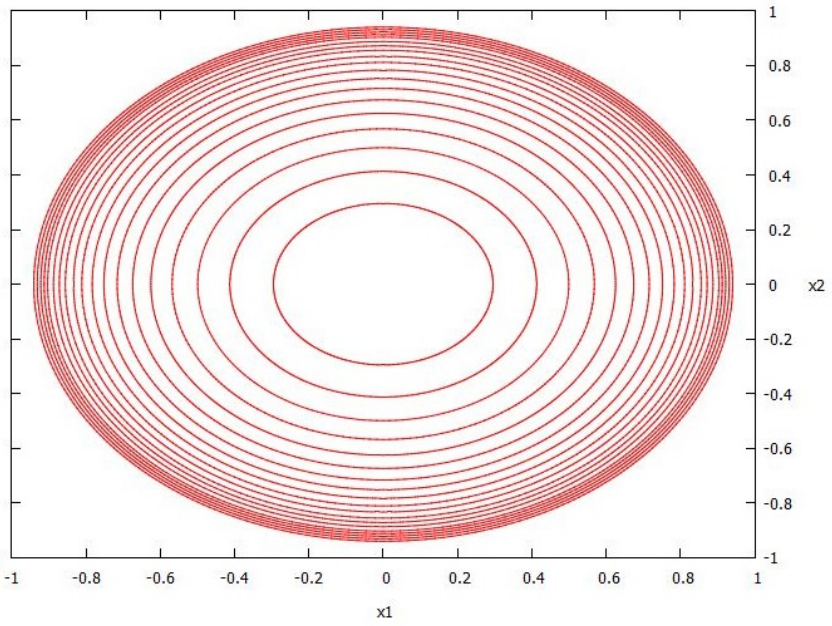

Fig. 5.

Some level curves of the CPA Lyapunov function from the 3. iteration for Example 2.

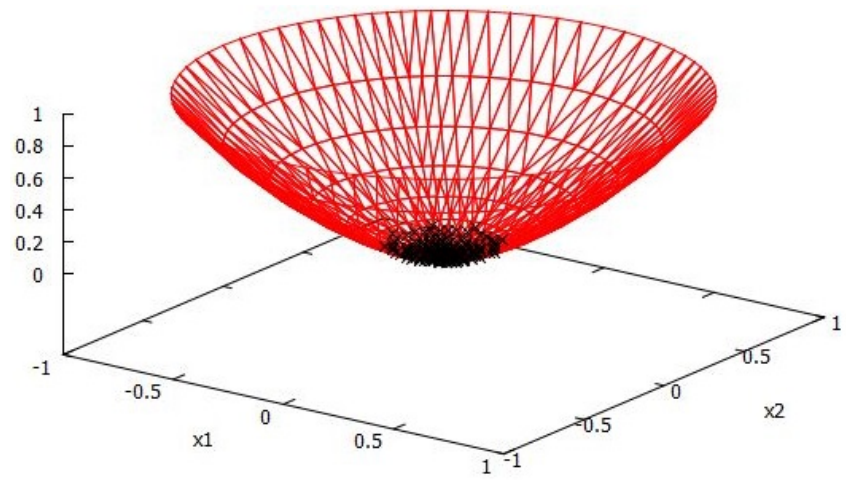

Fig. 6.

First iteration of the algorithm for the system in Example 3. We obtain a CPA Lyapunov function for the system with negative orbital derivative in 632 of the 800 simplices in the triangulation.

our method is noticeably slower than in the previous examples. We need for example six iterations of the algorithm to obtain a Lyapunov function with a negative orbital derivative on roughly $95 \%$ of the simplices, in which case the number of simplices has grown to a rather overwhelming 819, 200 . A smaller step-size for the RK4 integrator of the system does not improve the results. This slow convergence can be explained by the almost recurrent behavior of the system close to the origin. We plot the CPA Lyapunov function computed by the first two iterations of the algorithm in Figures 6 and 7. In Figure 8 we plot some level curves for the CPA Lyapunov function from the third iteration. In Table II we give two measures for the quality of the CPA Lyapunov functions computed in the different iterations. 


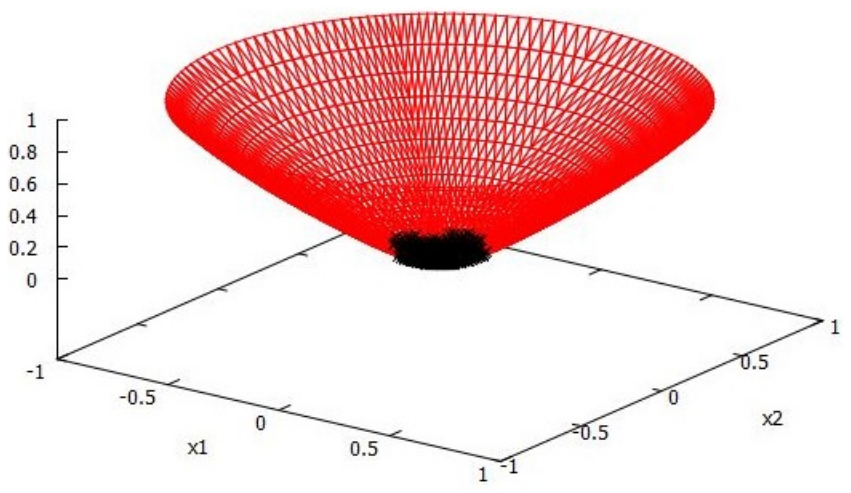

Fig. 7.

Second iteration of the algorithm for the system in Example 3. We obtain a CPA Lyapunov function for the system with negative orbital derivative in 2680 of the 3200 simplices in the triangulation.

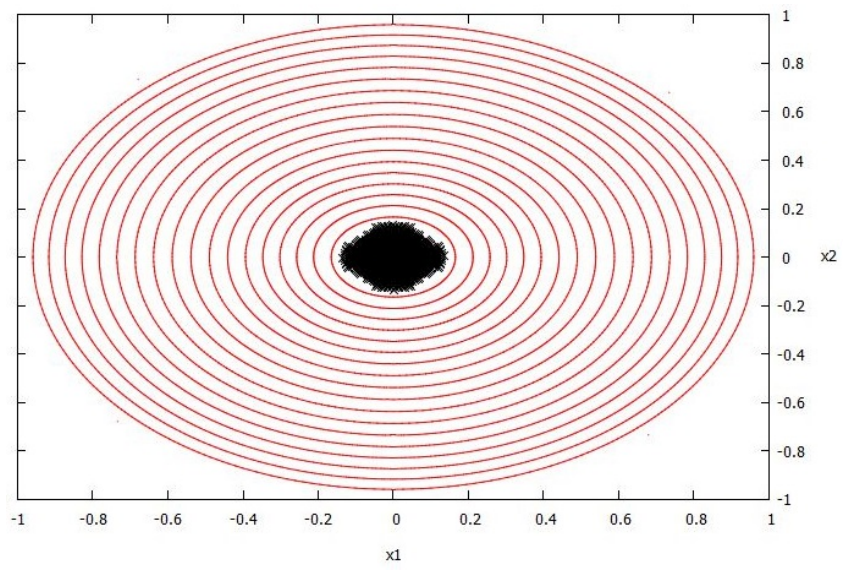

Fig. 8.

Some level curves of the CPA Lyapunov function from the third iteration for Example 3.

\begin{tabular}{|c|c|c|}
\hline Iteration & $\begin{array}{c}\text { Percentage of Simplices } \\
\text { with } D^{+} V(\mathbf{x})<0\end{array}$ & $\begin{array}{c}\text { Radius }(R) \text { Outside } \\
\text { Which } D^{+} V<0\end{array}$ \\
\hline 1 & $79.000 \%$ & 0.25000 \\
2 & $83.750 \%$ & 0.20250 \\
3 & $88.031 \%$ & 0.14063 \\
4 & $90.930 \%$ & 0.10563 \\
5 & $93.205 \%$ & 0.07563 \\
6 & $94.833 \%$ & 0.05790 \\
\hline
\end{tabular}

TABLE II

TWO DIFFERENT MEASURES FOR THE QUALITY OF THE COMPUTED CPA LYAPUNOV FUNCTIONS FOR THE DIFFERENT ITERATIONS. IN COLUMN 2 WE GIVE THE PERCENT OF THE NUMBER OF TRIANGLES $T_{i} \in \mathcal{T}$, WHERE THE ORBITAL DERIVATIVE $D^{+} V$ IS NEGATIVE AND IN COLUMN 3 WE GIVE THE RADIUS $R$ OF A BALL $B_{R}$, OUTSIDE OF WHICH THE ORBITAL DERIVATIVE IS NEGATIVE.

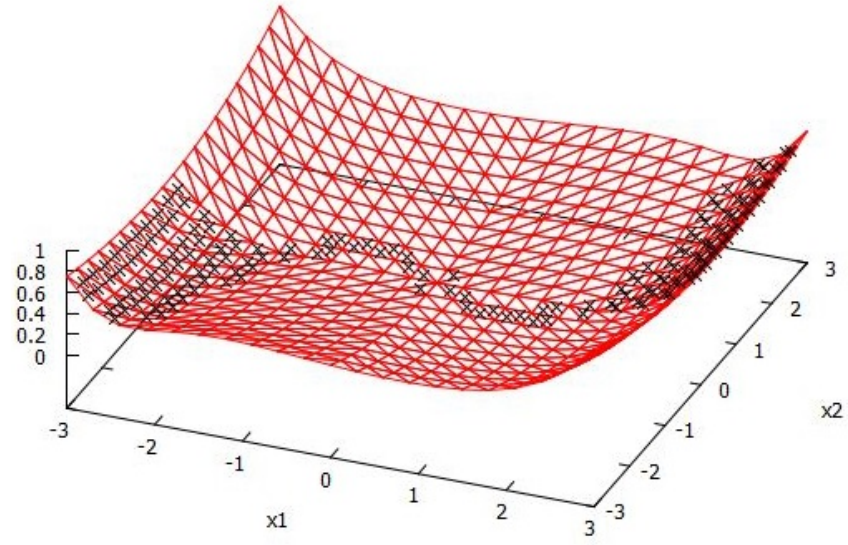

Fig. 9.

First iteration of the algorithm for the system in Example 4. We obtain a CPA Lyapunov function for the system with negative orbital derivative in 644 of the 800 simplices in the triangulation.

\section{Example 4}

Our last example is the following non-linear equation which we explore on the square $[-3,3]^{2}$.

$$
\begin{aligned}
& \dot{x}_{1}=x_{2} \\
& \dot{x}_{2}=-x_{1}+\frac{1}{3} x_{1}^{3}-x_{2} .
\end{aligned}
$$

In this example the origin is a stable equilibrium point, and we also have equilibrium points at $(\sqrt{3}, 0)$ and $(-\sqrt{3}, 0)$ that are unstable. Therefore we cannot expect to obtain a true Lyapunov function on the whole square, but as an interesting application we note that the domain in which we obtain a true Lyapunov function for the system gives a rough estimate on the basin of attraction for the stable equilibrium point at the origin. We plot the CPA Lyapunov function computed by the first two iterations of the algorithm in Figures 9 and 10. In Figure 11 we plot some level curves for the CPA Lyapunov function from the third iteration.

\section{CONCLUSIONS}

We have proposed a modified CPA method for the construction of Lyapunov functions on compact regions containing the origin using a classical Lyapunov function construction due to Massera. This modification has the benefit that computation of the vertex values for the candidate CPA Lyapunov function is much faster than the solving the linear program necessary for the original CPA method [15]. We presented several numerical examples demonstrating the utility of this modified CPA method.

While (II.3) provides a true Lyapunov function, the system trajectories obtained via the Runge-Kutta RK4 method are obviously an approximation. This causes no difficulty as we may, in fact, choose any values for the vertices to then define the candidate CPA Lyapunov function and then use Theorem 2.1 to determine whether or not those particular vertex values do, in fact, yield a negative orbital derivative 


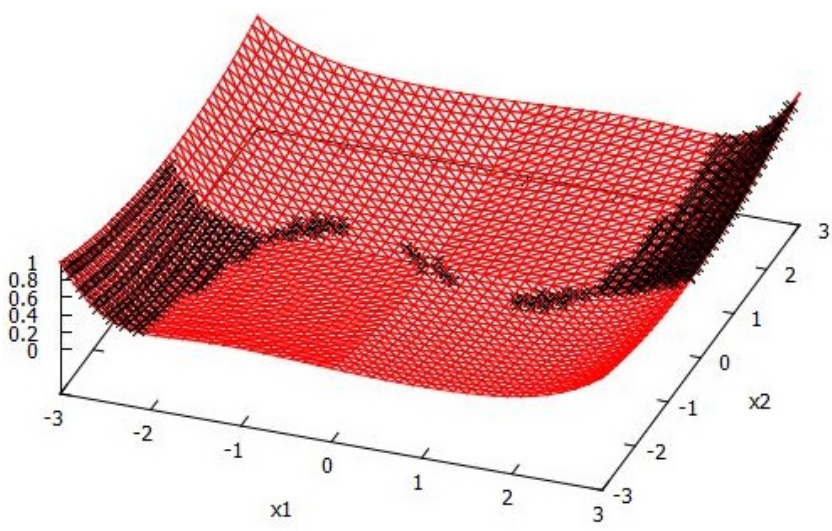

Fig. 10.

Second iteration of the algorithm for the system in example 4. We obtain a CPA Lyapunov function for the system with negative orbital derivative in 2598 of the 3200 simplices in the triangulation.

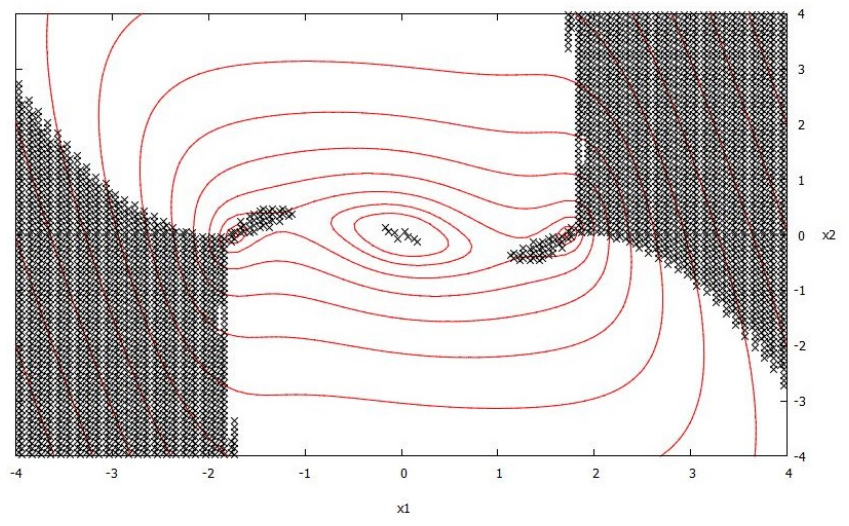

Fig. 11.

Some level curves of the CPA Lyapunov function from the 3. iteration for Example 4.

on each simplex. Using approximate values of (II.3) to fix the vertex values is then, in some sense, a principled guess since the results of [8] and [10] guarantee that, by a process of iteratively refining the triangulation, using exact values of (II.3) will eventually yield a CPA Lyapunov function.

\section{REFERENCES}

[1] R. Baier, L. Grüne, and S. Hafstein. Linear programming based Lyapunov function computation for differential inclusions. Discrete and Continuous Dynamical Systems Series B, 17:33-56, 2012.

[2] H. Ban and W. Kalies. A computational approach to Conley's decomposition theorem. Journal of Computational and Nonlinear Dynamics, 1-4:312-319, 2006.

[3] P. Giesl. Construction of Global Lyapunov Functions Using Radial Basis Functions. Number 1904 in Lecture Notes in Mathematics. Springer, 2007.

[4] P. Giesl and S. Hafstein. Construction of Lyapunov functions for nonlinear planar systems by linear programming. J. Math. Anal. Appl., 388:463-479, 2012.

[5] P. Giesl and S. Hafstein. Construction of a CPA contraction metric for periodic orbits using semidefinite optimization. Nonlinear Analysis: Theory, Methods \& Applications, 86:114-134, 2013.
[6] P. Giesl and S. Hafstein. Computation of Lyapunov functions for nonlinear discrete time systems by linear programming. J. Differ. Equ. Appl., 2014.

[7] P. Giesl and S. Hafstein. Revised CPA method to compute Lyapunov functions for nonlinear systems. J. Math. Anal. Appl., 410:292-306, 2014.

[8] S. Hafstein. A constructive converse Lyapunov theorem on exponential stability. Discrete Contin. Dyn. Syst., 10(3):657-678, 2004.

[9] S. Hafstein. An Algorithm for Constructing Lyapunov Functions. Electronic Journal of Differential Equations Mongraphs, 2007.

[10] S. Hafstein, C. M. Kellett, and H. Li. Continuous and piecewise affine Lyapunov functions using the Yoshizawa construction. In Proceedings of the American Control Conference, 2014.

[11] T. Johansen. Computation of Lyapunov functions for smooth nonlinear systems using convex optimization. Automatica, 36:1617-1626, 2000.

[12] W. Kalies, K. Mischaikow, and R. VanderVorst. An algorithmic approach to chain recurrence. Foundations of Computational Mathematics, 5-4:409-449, 2005.

[13] C. M. Kellett. A compendium of comparsion function results. In Press with Mathematics of Controls, Signals and Systems, February 2014.

[14] H. K. Khalil. Nonlinear Systems. Prentice Hall, 3 edition, 2002.

[15] S. Marinosson. Lyapunov function construction for ordinary differential equations with linear programming. Dynamical Systems, 17:137150, 2002.

[16] J. L. Massera. On Liapounoff's conditions of stability. Annals of Mathematics, 50:705-721, 1949.

[17] A. Papachristodoulou and S. Prajna. The construction of Lyapunov functions using the sum of squares decomposition. In Proceedings of the 41st IEEE Conference on Decision and Control, pages 3482-3487, 2002.

[18] M. Peet. Exponentially stable nonlinear systems have polynomial Lyapunov functions on bounded regions. IEEE Trans. Automatic Control, 54:979-987, 2009.

[19] M. Peet and A. Papachristodoulou. A converse sum-of-squares Lyapunov result: An existence proof based on the Picard iteration. In Proceedings of the 49th IEEE Conference on Decision and Control, pages 5949-5954, 2010.

[20] S. Ratschan and Z. She. Providing a basin of attraction to a target region of polynomial systems by computation of Lyapunovlike functions. SIAM J. Control and Optimization, 48(7):4377-4394, 2010.

[21] M. Rezaiee-Pajand and B. Moghaddasie. Estimating the region of attraction via collocation for autonomous nonlinear systems. Struct. Eng. Mech., 41(2):263-284, 2012.

[22] Z. She, H. Li, B. Xue, Z. Zheng, and B. Xia. Discovering polynomial Lyapunov functions for continuous dynamical systems. J. Symb. Comput., 58:41-63, 2013.

[23] E. D. Sontag. Comments on integral variants of ISS. Systems and Control Letters, 34(1-2):93-100, 1998.

[24] T. Yoshizawa. Stability Theory by Liapunov's Second Method. Mathematical Society of Japan, 1966. 